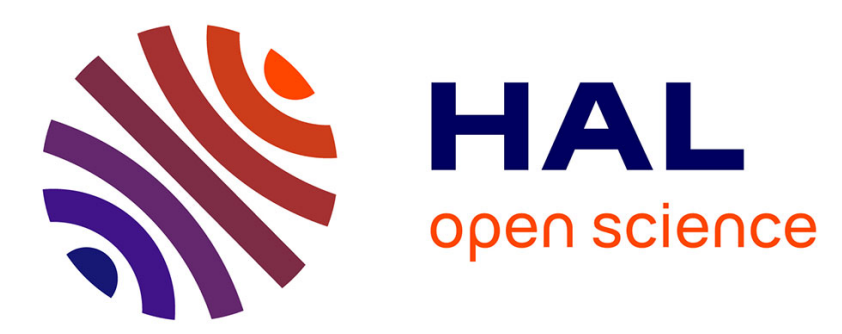

\title{
Relationship between biodiversity and agricultural production
}

Ilaria Brunetti, Mabel Tidball, Denis Couvet

\section{To cite this version:}

Ilaria Brunetti, Mabel Tidball, Denis Couvet. Relationship between biodiversity and agricultural production. 2018. halshs-01936005

\section{HAL Id: halshs-01936005 \\ https://shs.hal.science/halshs-01936005}

Preprint submitted on 27 Nov 2018

HAL is a multi-disciplinary open access archive for the deposit and dissemination of scientific research documents, whether they are published or not. The documents may come from teaching and research institutions in France or abroad, or from public or private research centers.
L'archive ouverte pluridisciplinaire HAL, est destinée au dépôt et à la diffusion de documents scientifiques de niveau recherche, publiés ou non, émanant des établissements d'enseignement et de recherche français ou étrangers, des laboratoires publics ou privés. 


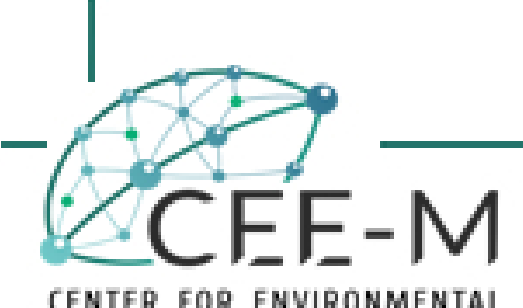

\title{
Relationship Between Biodiversity and Agricultural Production
}

\author{
Ilaria Brunetti, Mabel Tidball \& Denis Couvet
}

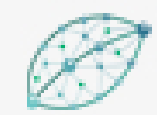

CEE-M Working Paper 2018-01 
Relationship Between Biodiversity and Agricultural Production
I. Brunetti, 1
M. Tidball $\stackrel{2}{\stackrel{2}{-}}$
and Denis Couvet ${ }^{3}$

${ }^{1}$ École Polytechnique, Paris-Saclay, Muséum Nationale d'Histoire Naturelle, Paris, France ${ }^{2}$ CEE-M; Univ. Montpellier, CNRS, INRA, SupAgro, Montpellier, France

${ }^{3}$ Muséum Nationale d'Histoire Naturelle, Paris, France 


\begin{abstract}
Agriculture is one of the main causes of biodiversity loss. In this work we model the interdependent relationship between biodiversity and agriculture on a farmed land, supposing that, while agriculture has a negative impact on biodiversity, the latter can increase agricultural production. Farmers act as myopic agents, who maximize their instantaneous profit without considering the negative effects of their practice on the evolution of biodiversity. We find that a tax on inputs can have a positive effect on yield since it can be considered as a social signal helping farmers to avoid myopic behavior in regards to the positive effect of biodiversity on yield. We also prove that, by increasing biodiversity productivity the level of biodiversity at equilibrium decreases, since when biodiversity is more productive farmers can maintain lower biodiversity to get the same yield.
\end{abstract}

\title{
1 Introduction
}

The decline of biodiversity observed on farmed land in recent years is nowadays a major concern Butchart et al. (2010). Besides spatial extension of agricultural landscapes, the intensification of farming practices is considered as one of the major causes of biodiversity loss and its consequences affect the whole ecosystem. At the opposite, biodiversity is necessary to agriculture, at least through provisioning and regulating services MEA (2005). As a result, one could determine an optimal level of agricultural intensification, maximizing an agricultural indicator (yield, farmers' revenue, ... ), possibly a biodiversity level (the more biodiversity is valued, lower the optimal level of agriculture intensification).

Despite the growing interest in the effects of agriculture on the surrounding environment and the extensive literature dealing with these issues, there's still a lack of quantitative models and analytical results. Furthermore, while the damages caused by agriculture to biodiversity are widely studied, only small work has been done on the positive effects that the preservation of biodiversity may have on farming activity. In Desquilbet et al. (2013) the authors develop an economic model to compare the impacts of intensive versus extensive agriculture (land sparing vs. land sharing) on biodiversity and on welfare and they define biodiversity per unit of land as a decreasing function of yield. In their model biodiversity is static and production is independent from the level of biodiversity, while it has been observed that biodiversity may provide important services for farming activity, as soil quality Durand et al. (2017) and pollinators Garibaldi et al. (2011).

In this work we model the interdependent relationship between agriculture and biodiversity. 
Biodiversity is defined as a dynamic process depending on agricultural production and on the use of pesticides, while yield is increased by biodiversity. Farmers control the amount of inputs and labor to be employed for production, where inputs are taxed; they maximize their profit without taking into account the effect of their practices on biodiversity. In this framework, where biodiversity and yield are interdependent, we analyze the effect of the tax on biodiversity and agriculture. We distinguish between local and after migration biodiversity, in order to capture the effect of spatial migration occurring among different farmed lands Nishimura and Kishida (2001).

The paper is organized as follows: after presenting the general model in Section 2, in Section 3 we provide the formula for biodiversity dynamics. We then introduce in Section 4 the farmers profit optimization problem and we compute the optimal controls. In Section 5 we determine the optimal stationary state of local biodiversity dynamic, proving the existence and the uniqueness of the solution, and we present explicit formulas for two particular cases. In Section 6 we analyze the effect of the tax on biodiversity and on farmers behavior at the optimal stationary state for the two particular cases. In Section 7 we illustrate some numerical examples.

\section{The General Model}

We consider a farmed region equally divided among a finite number of farmers $N \geq 2$, so that each of them disposes of an unit of land. Farmers can choose the amount of chemical inputs to be used, denoted by $A_{i} \in[0,1]$ and labor $\ell_{i} \in[0,1]$. On one hand agricultural production has a negative impact on biodiversity: in particular, we suppose that agricultural production and chemical inputs affect the carrying capacity of the land to host biodiversity. Whatever technique is used for agricultural production, even when only small quantities of pesticides and fertilizers are used, still farming activity damages biodiversity, since the land is devoted to humans and not to biodiversity Newbold et al. (2015), Durand et al. (2017). On the other hand, biodiversity also has an impact on agricultural production (or yield, since in our case they coincide). We assume that the level of biodiversity on farmer's $i$ land not only depends on the choices of farmer $i=1, \ldots N$, but also on the biodiversity in the others land, due to the biodiversity's migration effect, such that biodiversity moves, from one land to the other within the same land $i=1, \ldots N$. 


\section{Biodiversity and Yield Equations}

\subsection{Effects of migration on biodiversity dynamics}

We denote by $B_{i}^{t} \in[0,1]$ the local biodiversity on land $i$ at time $t=0,1, \ldots$. Since biodiversity can move, from one land to the other (think at birds, insects ...) we introduce the after migration biodiversity, $\tilde{B}_{i}^{t} \in[0,1]$. After migration biodiversity is the biodiversity which can be observed on a farmer's land after this shift of biodiversity among the different lands:

$$
\tilde{B}_{i}^{t}:=\sum_{j=1}^{N} m_{i j} B_{j}^{t}, \quad \sum_{j=1}^{N} m_{i j}=1 .
$$

The coefficient $m_{i i}$ represents the migration effect within the same land $i$, while $m_{i j}$, with $j \neq i$ from land $j$ to $i$.

\subsection{Dependence of Yield on Biodiversity}

The yield produced by farmer $i$ on his area of land depends on the amount of inputs used $A_{i} \in[0,1]$, on labor $\ell_{i} \in[0,1]$ and on the after migration biodiversity. At each instant of time $t$, we have that, $\forall i=1, \ldots, N$ :

$$
Y_{i}^{t}:=\beta_{i} v_{i}\left(\tilde{B}_{i}^{t}\right)\left(\ell_{i}^{t}\right)^{\nu_{i}}\left(A_{i}^{t}\right)^{\eta_{i}}, \quad \beta_{i}>0, \forall i
$$

with $\eta_{i}+\nu_{i}<1$. This is a Cobb-Douglas production function with decreasing return to scale, where the production factors considered are inputs and labor and productivity is a function of biodiversity. While in standard Cobb-Douglas functions productivity is usually constant, here we suppose that it is a function of $\tilde{B}_{i}^{t},\left(v_{i}\left(\tilde{B}_{i}^{t}\right)\right.$, where $v_{i}$ is increasing in $\left.\tilde{B}_{i}^{t}\right)$. It can be taught as the ability of farmer to employ biodiversity on his land. For sake of computation, here, we define $v_{i}$ as follows:

$$
v_{i}\left(\tilde{B}_{i}\right):=\tilde{B}_{i}^{\gamma_{i}}
$$

which leads to

$$
Y_{i}^{t}=\beta_{i}\left(\tilde{B}_{i}^{t}\right)^{\gamma_{i}}\left(\ell_{i}^{t}\right)^{\nu_{i}} \times\left(A_{i}^{t}\right)^{\eta_{i}}
$$


After migration biodiversity $\tilde{B}_{i}$ is not a production factor in a strict sense, because it's not directly controlled by the farmer. Note that, since $\tilde{B}_{i}, \ell_{i}, A_{i}$ are in the interval $[0,1]$, for given values of these variables, the higher are the exponents $\gamma_{i}, \nu_{i}$ and $\eta$, the lower is $Y_{i}^{t}$ and each factor's efficiency is a decreasing function of its exponent.

\subsection{Biodiversity dynamics, depending on farming practices, density-dependence and migration}

In our model, there are three different phenomena affecting biodiversity. Farming practices, densitydependence -due to limited resources- and migration. For the sake of mathematical tractability, we chose to consider conjointly these three effects.

Local biodiversity evolves on time according to a continuous-time dynamic equation that takes into account in one hand the biological effect of density dependence and migration and in the other hand farming activities:

$$
\dot{B}_{i}=R \tilde{B}_{i}\left(1-\frac{\tilde{B}_{i}}{M_{i}\left(A_{i}, Y_{i}\right)}\right)=R \sum_{j=1}^{N} m_{i j} B_{j}\left(1-\frac{\sum_{j=1}^{N} m_{i j} B_{j}}{M_{i}\left(A_{i}, Y_{i}\right)}\right), \quad i=1, \ldots, N
$$

where $R>0$ is the intrinsic growth rate of local biodiversity and $M_{i}$ represents the carrying capacity of land $i$ to host biodiversity at time $t$, which depends the level of inputs used $A_{i}$ and on yield $Y_{i}$, according to the following linear function:

$$
M_{i}\left(A_{i}, Y_{i}\right):=a_{i}-b_{i}\left(\alpha A_{i}+Y_{i}\right), \quad \alpha \geq 0 .
$$

The parameters $a_{i}$ and $b_{i}$ characterize each land $i$ and its reaction to the farming activity, while the parameter $\alpha$ characterizes the specific effect of inputs on biodiversity. As a matter of fact, farming activity impacts biodiversity both through production -no matter how this production is obtained due to exported biomass and through agricultural practices (chemical inputs, fetilizers), since, independently on the amount of yield obtained, when inputs like pesticides and fertilizers are used, they have a negative impact on biodiversity Durand et al. (2017). When $M$ is constant, the evolution of the biodiversity follows an usual differential equation with migrations Nishimura and Kishida (2001). In Mouvsset et al. (2013) $M$ is not constant, it depends on the share of land dedicated to agriculture; in Bluthgena et al. (2012) the authors define an index of pressure on biodiversity due to land use, which summarizes the effects of chemical fertilizers, mowing and livestock grazing. 


\section{Farmers' Behavior: Profit Function and Optimal Strategies}

The instantaneous profit functions of a farmer $i$ (i.e. the profit the farmer obtains at time $t$ ) is given by a standard function:

$$
\pi_{i}^{t}=p Y_{i}^{t}-w_{A} A_{i}^{t}-w_{\ell} \ell_{i}^{t}-\tau A_{i}^{t}
$$

where $p$ is the fixed price, $w_{A}$ and $w_{\ell}$ represent the cost per unit of inputs and labor respectively and $\tau$ is a fixed tax. Farmers are supposed to choose the quantity of inputs to be used and the amount of labor to employ in order to maximize their profit, while they are not aware of the impact of their choices on biodiversity, since the level of biodiversity and the effects of agriculture are very complex to be quantified by a single farmer. When the information available to the decision-makers are limited, it is said that they adopt a myopic behavior, as the individual has a narrow visibility of the entire system. Precisely, the farmer does not infer the evolution of biodiversity and the impact it could have on future yield.

In our case this means that, when farmer $i$ maximizes his instantaneous profit function $\pi_{i}^{t}$, after migration biodiversity $\tilde{B}_{i}^{t}$ is assumed to be fixed and given, which leads to an optimization problem, where, at each instant of time $t$ farmer $i$ maximizes $\pi_{i}^{t}$ w.r.t. the controls, $A_{i}^{t}$ and $\ell_{i}^{t}$. Then, by imposing $\frac{\partial \pi_{i}^{t}}{\partial A_{i}^{t}}=0$ and $\frac{\partial \pi_{i}^{t}}{\ell_{i}^{t}}=0$, we obtain the optimal controls:

$$
\left\{\begin{array}{l}
A_{i}^{t *}=K_{A_{i}}\left(\tilde{B}_{i}^{t}\right)^{\theta}, \quad \theta:=\frac{\gamma_{i}}{1-\eta_{i}-\nu_{i}} \\
\ell_{i}^{t *}=K_{\ell_{i}}\left(\tilde{B}_{i}^{t}\right)^{\theta}
\end{array}\right.
$$

where $K_{A_{i}}:=\left[p \beta_{i} \eta_{i}^{1-\nu_{i}} \nu_{i}^{\nu_{i}}\left(w_{A}+\tau\right)^{-\left(1-\nu_{i}\right)} w_{\ell}^{-\nu_{i}}\right]^{\frac{1}{1-\eta_{i}-\nu_{i}}}$, and $K_{\ell_{i}}:=\left[p \beta_{i} \eta_{i}^{\eta_{i}} \nu_{i}^{1-\eta_{i}}\left(w_{A}+\tau\right)^{-\eta_{i}} w_{\ell}^{-\left(1-\eta_{i}\right)}\right]^{\frac{1}{1-\eta_{i}-\nu_{i}}}$.

When farmer $i$ adopt the optimal strategies at time $t$, he obtain the following instantaneous optimal yield:

$$
Y_{i}^{t *}=v_{i}\left(\tilde{B}_{i}^{t}\right)\left(A_{i}^{t *}\right)^{\eta_{i}}\left(\ell_{i}^{t *}\right)^{\nu_{i}}=K_{Y_{i}} \tilde{B}_{i}^{\theta}
$$

where: $K_{Y_{i}}:=\beta K_{A_{i}} K_{\ell_{i}}=\left(\beta_{i} p^{\nu_{i}+\eta_{i}} \eta_{i}^{\eta_{i}} \nu_{i}^{\nu_{i}}\left(w_{A}+\tau\right)^{-\eta_{i}} w_{\ell}^{-\nu_{i}}\right)^{\frac{1}{1-\eta_{i}-\nu_{i}}}$. The corresponding optimal profit at time $t$ is:

$$
\pi_{i}^{t *}=\left(\tilde{B}_{i}^{t}\right)^{\theta}\left(p K_{Y_{i}}-\left(w_{A}+\tau\right) K_{A_{i}}-w_{\ell} K_{\ell}\right)=K_{\pi_{i}}\left(\tilde{B}_{i}^{t}\right)^{\theta}
$$


where $K_{\pi_{i}}:=p K_{Y_{i}}-\left(w_{A}+\tau\right) K_{A_{i}}-w_{\ell} K_{\ell}$.

\section{Stationary State of Biodiversity}

When considering this myopic optimal behavior of each farmer, local biodiversity evolves on time according to the dynamic equation (44), with the controls computed at the optimal level $A_{i}^{*}$ and $\ell_{i}^{*}$ :

$$
\dot{B}_{i}=R \tilde{B}_{i}\left(1-\frac{\tilde{B}_{i}}{M\left(A_{i}^{*}, Y_{i}^{*}\right)}\right), \quad B_{i}(0)=B_{i 0}, \quad \forall i=1, \ldots N .
$$

We are interested at the stationary state of local biodiversity. When $t \rightarrow \infty$, (5) converges to an optimal stationary state, denoted by $B_{i}^{\infty *}$, which is obtained by solving $\forall i=1, \ldots N$ :

$$
\dot{B}_{i}=R \tilde{B}_{i}\left(1-\frac{\tilde{B}_{i}}{a_{i}-b_{i}\left(\alpha A_{i}^{*}+Y_{i}^{*}\right)}\right)=R \tilde{B}_{i}\left(1-\frac{\tilde{B}_{i}}{a_{i}-b_{i} K_{i}\left(\tilde{B}_{i}\right)^{\theta}}\right)=0
$$

where

$$
K_{i}:=\alpha K_{A_{i}}+K_{Y_{i}}, \quad \theta=\frac{\gamma}{1-\nu_{i}+\eta_{i}} .
$$

Note that, if we look at the internal stationary points, that is the non-null solutions of (10), we obtain $N$ independent equations in terms of after migration biodiversity $\tilde{B}_{i}: 1-\frac{\tilde{B}_{i}}{a_{i}-b_{i} K_{i}\left(\tilde{B}_{i}\right)^{\theta}}=0$, $i=1, \ldots, N$.

Proposition 1 Existence and uniqueness of the stationary state $\tilde{B}_{i}^{\infty *}$. If $a-b_{i} K_{i}<1$, then there exists a unique solution $\left.\tilde{B}_{i}^{\infty *} \in\right] 0,1[$.

Proof. See Appendix A.

Proposition 2 Stationary State and Productivity as a Function of $\gamma$ When optimal strategies are used by farmers, at the stationary state we obtain that:

- $\frac{\partial \tilde{B}_{i}^{\infty *}}{\partial \gamma}>0$, and thus, after migration biodiversity increases with $\gamma$.

- $\frac{\partial v\left(\tilde{B}_{i}^{\infty *}\right)}{\partial \gamma}<0$ which means that, by increasing $\gamma$, the productivity of after migration biodiversity decreases;

Proof. See Appendix A. 
This proposition means that increasing biodiversity productivity -in regards to yield - has the counter-intuitive effect that the level of biodiversity at equilibrium decreases. The reason being that farmers can maintain lower biodiversity to get the same yield, when biodiversity efficiency increases.

Remark 1 The behavior of the stationary state $\tilde{B}_{i}^{\infty *}$ with respect to the parameters of the model As expected, we find that if the carrying capacity of the land increases (or decreases), then $\tilde{B}_{i}^{\infty *}$ also increases (resp. decreases). Looking at the behavior of optimal stationary biodiversity w.r.t. the economic parameters we have that $\tilde{B}_{i}^{\infty *}$ is increasing with the costs of inputs and labor, while it decreases with price: as a matter of fact, if prices increases or costs decrease farmers produce more, which affects biodiversity. These results are proved mathematically in Appendix A

\subsection{Stationary State of $B_{i}^{\infty *}$}

Proposition 3 Existence and uniqueness of stationary $B_{i}^{\infty *}$. Once the system is solved in terms of $\tilde{B}_{i}^{\infty *}$, the stationary solution in terms of local biodiversity $B_{i}^{\infty *}$, can be obtained by solving the linear system $\sum_{j=1}^{N} m_{i j} B_{i}^{\infty *}=\tilde{B}_{i}^{\infty *}, \forall i=1, \ldots N$. The solution is unique if and only if the matrix of coefficients has rank $N$.

Remark 2 We can study two particular cases, which allow us to compute optimal stationary local biodiversity as a function of after migration biodiversity.

(i) We suppose that $N=2$, i.e. that there are only two farmers sharing the farmed region. Local biodiversity at the optimal stationary state is given by:

$$
B_{i}^{\infty *}=\frac{\tilde{B}_{i}^{\infty *} m_{i j}-\tilde{B}_{j}^{\infty *} m_{j j}}{m_{i i} m_{j j}-m_{i j} m_{j i}} .
$$

(ii) If the region considered is small, then we can suppose that all the migration rates from a land $j$ to a different land $i, i, j=1, \ldots N$, with $i \neq j$, are equals and that each local biodiversity has the same effect on itself, that is:

$$
\forall i, \forall j \neq i \quad m_{i i}=1-m \quad m_{i j}=\frac{m}{N-1}
$$

In this case, the internal stationary points of the optimal local biodiversity dynamics are given 
by:

$$
B_{i}^{\infty *}=\frac{1}{N(1-m)-1}\left((N-1-m) \tilde{B}_{i}^{\infty *}-m \sum_{j \neq i} \tilde{B}_{j}^{\infty *}\right)
$$

(iii) Note that, in the latter case, if the farmers are completely symmetric, i.e. $a_{i}=a, b_{i}=b$, $\beta_{i}=\beta, \forall i=1, \ldots, N$, then

$$
B_{i}^{\infty *}=\tilde{B}_{i}^{\infty *}=B^{\infty *}, \quad \forall i=1 \ldots N
$$

that is, local and after migration biodiversity coincide and are the same for all the farmers.

See Appendix $B$ for the proofs of these inequalities.

\section{The Effects of the Tax at Stationary State}

We now study the impact of the tax $\tau$ imposed on inputs on the optimal stationary solutions of our model. Proposition (5) is proved for the two particular cases presented in Remark 2 of the previous section, while the other results are general.

\subsection{Tax and Biodiversity}

Proposition 4 After migration biodiversity and tax We have that:

$$
\frac{\partial \tilde{B}_{i}^{\infty *}}{\partial \tau}>0, \quad \forall i=1, \ldots, N
$$

which means that, by increasing the tax $\tau$, optimal after migration biodiversity at the stationary state always increases.

Proof. See Appendix C.1.

This means that, as expected, the tax on inputs has a positive effect on biodiversity level at the stationary state: the higher is the tax, the higher is $\tilde{B}_{i}^{\infty *}$.

Proposition 5 Local migration biodiversity and tax For local biodiversity, the behavior at the stationary state is not fixed, that is, local biodiversity can be increasing or decreasing w.r.t. the $\operatorname{tax}$. 
Proof. See Appendix C.2.

Proposition 6 Optimal solutions and tax The behavior of the optimal stationary quantities $A_{i}^{\infty *}, \ell_{i}^{\infty *}$ and $Y_{i}^{\infty *}$ and $\pi^{\infty *}$ with respect to $\tau$ are not fixed, except for the following particular cases.

- If agricultural production impacts the carrying capacity only through the yield and not through the inputs used, then yield decreases with the tax, i.e.:

$$
\alpha=0 \Rightarrow \frac{\partial Y_{i}^{*}}{\partial \tau}<0
$$

. Furthermore, if $\alpha=0$ and $\eta<1 / 2$ then optimal labor also decreases with the tax $\left(\frac{\partial \ell_{i}^{*}}{\partial \tau}<0\right)$.

- If yield and inputs have no impact on the carrying capacity $M$, or if biodiversity doesn't impact agricultural production, then optimal yield, input and labor's levels decrease with the tax:

$$
b=0 \quad O R \quad \gamma=0 \Rightarrow\left\{\begin{array}{c}
\frac{\partial Y_{i}^{*}}{\partial \tau}<0 \\
\frac{\partial A_{i}^{*}}{\partial \tau}<0 \\
\frac{\partial \ell_{i}^{*}}{\partial \tau}<0
\end{array}\right.
$$

Proof. See Appendix C.3.

The tax contributes to avoid the negative effect of the myopic behavior of the farmer, forcing farmers to benefit from the positive impact of biodiversity on yield.

\section{Some Numerical Examples}

\subsection{Stationary State of Biodiversity and Productivity}

We consider two farmers, with two different biodiversity productivity coefficients, with $\beta_{1}=$ $1.2, \beta_{2}=0.8$, so that for farmer one biodiversity is more productive in agricultural terms. We set the following values of the other parameters: $a_{i}=1, \alpha=1, b=1, \eta_{i}=0.4, \nu_{i}=0.4, p=2, \tau=$ $1, w_{A}=1, w_{\ell}=2, i=1,2$. In figure 1 we show that, as stated in Proposition 2 after migration biodiversity $\tilde{B}_{i}^{\infty *}$ is decreasing in $\gamma$, while its productivity $v\left(\tilde{B}_{i}^{\infty *}\right)$ decreases. Note that, due to higher production, farmer 1 has a significantly lower value of $\tilde{B}_{1}^{\infty *}\left(\right.$ and $\left.v\left(\tilde{B}_{1}^{\infty *}\right)\right)$, compared to $\tilde{B}_{2}^{\infty *}$ $\left(\operatorname{resp} v\left(\tilde{B}_{2}^{\infty *}\right)\right)$. 


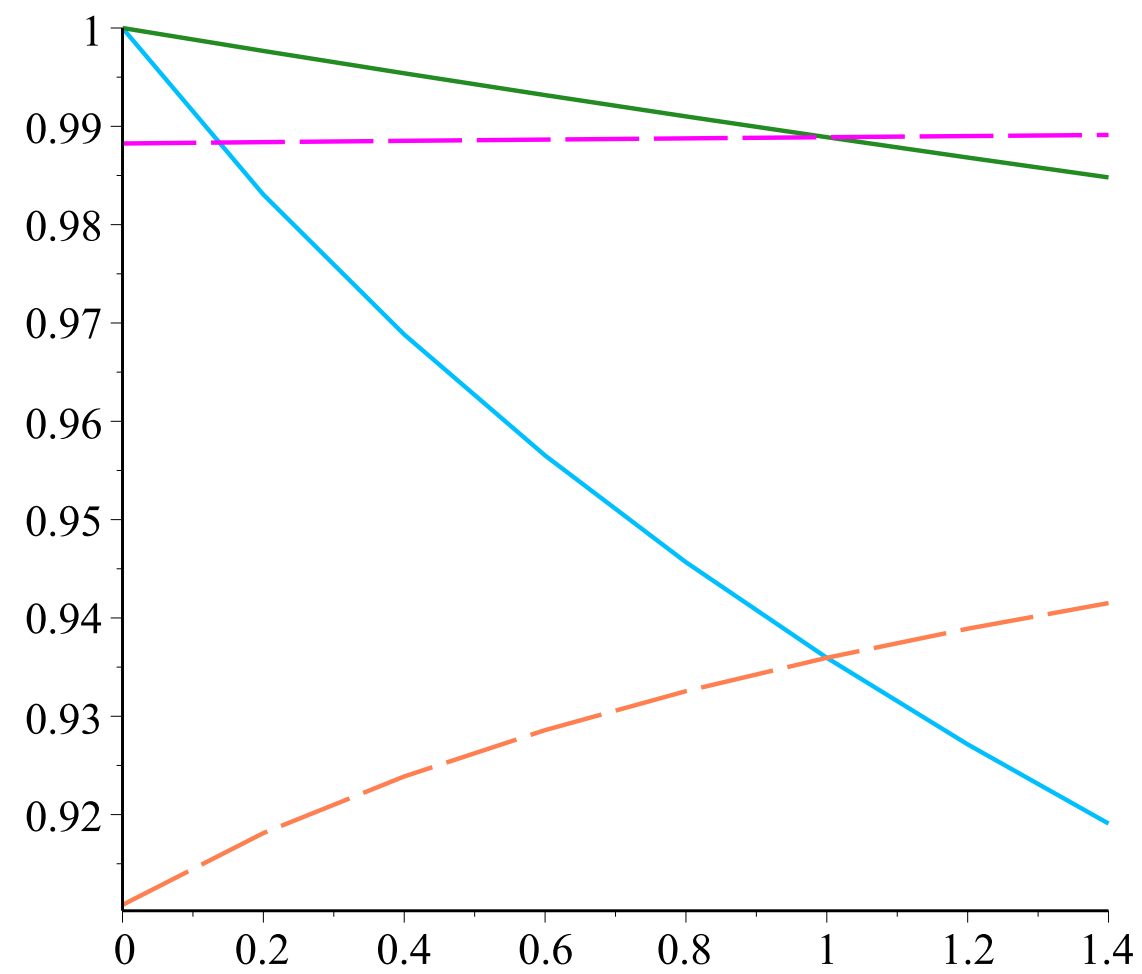

\section{Productivity $1--$ Btilde $1-$ Productivity 2}

- Btilde 2

Figure 1: $\tilde{B}_{i}^{\infty *}$ and $v\left(\tilde{B}_{i}^{\infty *}\right)$ as a function of $\gamma \in[0,1.4], i=1,2$. As proved in Proposition 2 , $\frac{\partial \tilde{B}_{i}^{\infty *}}{\gamma}>0$, while $\frac{\partial v\left(\tilde{B}_{i}^{\infty *}\right)}{\gamma}<0$.

\subsection{Stationary States and the Tax}

We now study the behavior of the optimal stationary solutions as a function of $\tau$, in order to show the impact of the tax on biodiversity and on farmer's behavior. In particular, we first show that, as stated in Proposition 4 and 5, while after migration biodiversity is always increasing with the tax, local biodiversity may decrease. Then, in Subsection 7.2.2 we illustrate a case where the tax has a positive effect not only in increasing biodiversity and decreasing inputs, but also on agricultural yield and farmer's profit. As a matter of fact, as presented in Proposition 6, we find that, while in the standard case, where biodiversity doesn't enter in the production function, the tax has always a negative impact on yield and profit, when we consider the productivity of biodiversity, it changes the behavior of yield. 


\subsubsection{Example: local biodiversity may decrease with the tax}

We consider two farmers with a different productivity coefficient $\beta_{1}=0.8, \beta_{2}=0.6$, while all the other parameters are the same for the two farmers: $a=0.8, \eta=\nu=0.4, \gamma=0.2$ (which implies $\theta=1), R=1, p=2, w_{A}=1, w_{\ell}=2, m=0.4$. We study the behavior of optimal stationary biodiversity w.r.t. the $\operatorname{tax} \tau \in[0,1]$.

As stated in Proposition 4, we observe in 2 that, for both farmers $\tilde{B}_{i}^{\infty *}, i=1,2$ increases with the tax, while for farmer $1, B_{1}^{\infty *}$ is decreasing in $\tau$ : as we observed in Proposition [5, the behavior of $B_{i}^{\infty *}$ w.r.t. the tax is not fixed.

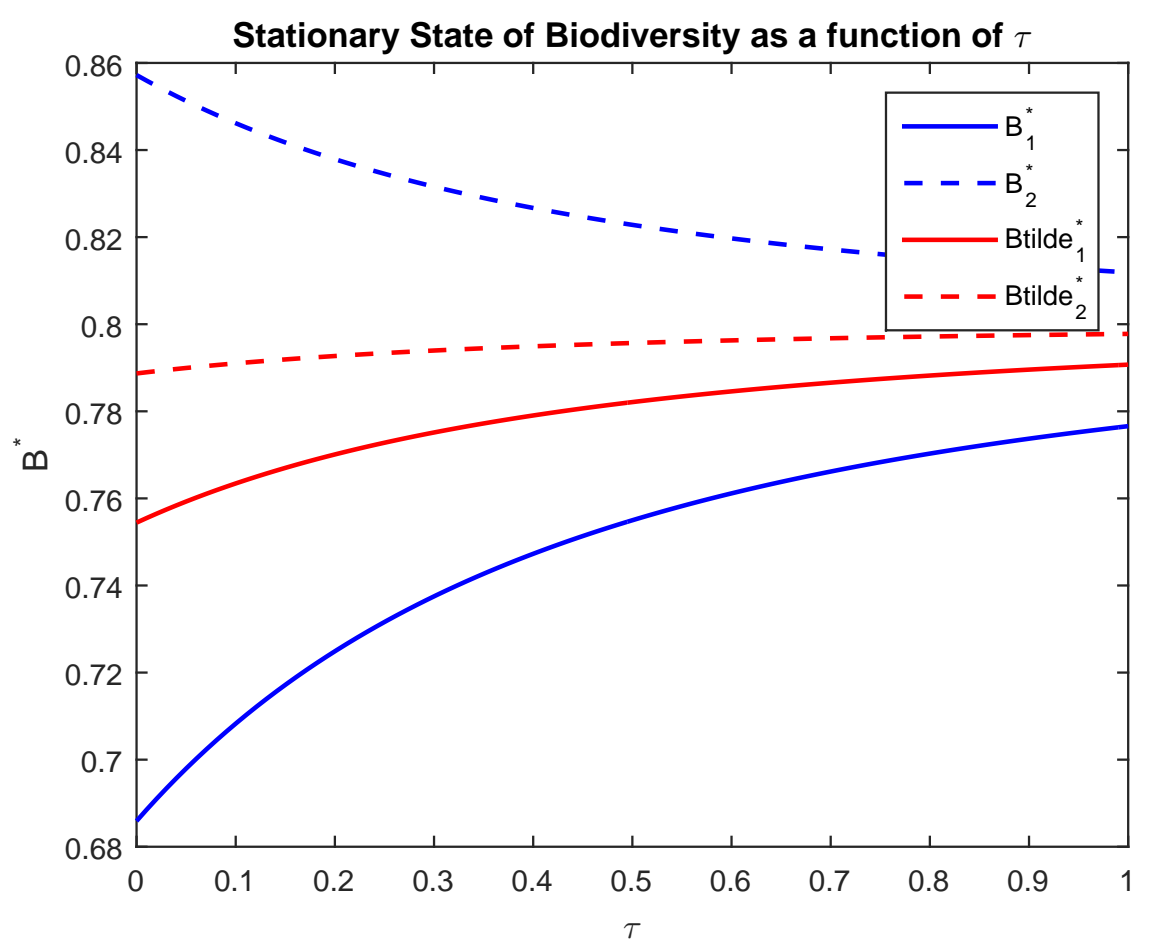

Figure 2: $\tilde{B}_{i}^{\infty *}$ and $B_{i}^{\infty *}$ as a function of $\tau, i=1,2$. Note that, for farmer 2, local biodiversity $B_{2}^{\infty *}$ (dotted blue line) decreases with $\tau$.

\subsubsection{Example: yield and profit can increase with the tax}

We consider two farmers, with a different exponent $\gamma_{i}: \gamma_{1}=0.4$ and $\gamma_{2}=0.8$ (and thus $\theta_{1}=1$, $\left.\theta_{2}=2\right)$. We then set $a=1, b=1, R=1, p=5, \alpha=1, \beta=1, w_{\ell}=2, w_{A}=1, \eta=\nu=0.3 ; m=0.1$. Even if the optimal level of inputs decreases with the tax, we can observe in figure 3 that yield and profit of farmer 1 are increasing in $\tau \in[0,1]$ : as we proved in Proposition 6 , the behavior of the 
optimal solutions w.r.t. $\tau$ are not fixed, except for two particular cases.

We also observe that $A_{1}^{\infty *}>A_{2}^{\infty *}$ and $B_{1}^{\infty *}<B_{2}^{\infty *}$ : since after migration biodiversity is more productive for farmer 1 , then he needs less biodiversity than farmer 2 , he's less dependent on it and he can uses more inputs.

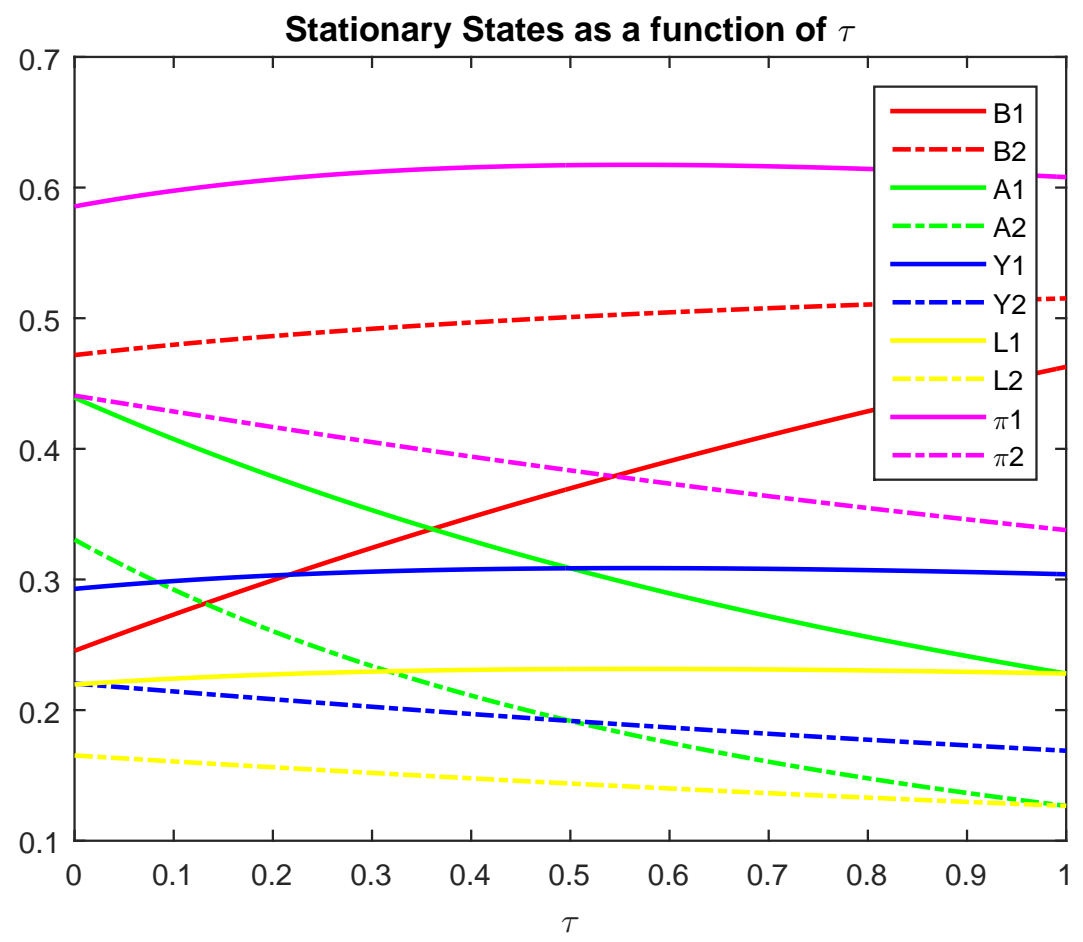

Figure 3: The optimal stationary values of the controls, after migration biodiversity and profit plotted as a function of the tax tau. Note that, for farmer one (continuous line), profit and yield increase with $\tau$.

\section{Conclusions}

In this work we presented a model to describe the interdependent relationship between farming activity and biodiversity on farmed land where, besides considering the negative impact of agriculture on biodiversity, we also supposed that biodiversity increases production. We defined local and after migration biodiversity, in order to consider the phenomenon of spatial migration of biodiversity among nearby lands and we define their evolution on time. We considered a finite number of farmers who control the level of inputs and labor, while they are myopic with respect to biodiversity, i.e. they don't consider the effects of their practices on the environment.

We computed the stationary state of biodiversity dynamics when farmers adopt their opti- 
mal strategies and we studied the impact of the tax on the optimal solutions. First of all, we proved the existence and the uniqueness of the stationary states $\tilde{B}_{i}^{\infty *}$ and $B_{i}^{\infty *}$ and we showed that $B_{i}^{\infty *}$ and $\tilde{B}_{i}^{\infty *}$ may have different behaviors w.r.t. the tax.

Two original results arose, due to our hypotheses that biodiversity has positive effects on yield, but that there is time delay in regards to the positive effect of biodiversity on agricultural yield, compared to the effect of agriculture on biodiversity, leading to a progressive adjustment of biodiversity to agricultural activity.

First, the tax can have positive effect on yield because the tax can be considered as a social signal helping farmers to avoid myopic behavior in regards to the positive effect of biodiversity on yield, discounting its future positive effect, as such positive effect is not immediate.

Second, we proved that, by increasing biodiversity productivity the level of biodiversity at equilibrium decreases, since when biodiversity is more productive farmers can maintain lower biodiversity to get the same yield. Our result brings attention to the fact that ecological engineering, associated to ecological intensification, might have detrimental impact on biodiversity, might not be favorable to the maintenance of biodiversity, at the opposite of what is often assumed (e.g. Cordonnier and Peyron (2015) and Geertsema et al. (2016)). Another consequence is that it brings attention to differences between the ends of preservation of biodiversity and ecosystems services. That is our results show that preserving ecosystem services can be detrimental to biodiversity when biodiversity is made more efficient in the provision of ecosystem services.

There are different possible direction for future developments of our model. It would be interesting to further investigate the regulation issue, by introducing a non-myopic regulator as a player of the game. The regulator should maximize a utility function where biodiversity is taken into account, which would leads to a two step game. We could also consider and compare other regulation instruments to preserve biodiversity, as an alternative or in addition to the tax, as, for examples labels. By labeling organic products, one could encourage consumers to choose organic products, which could possibly orientate agriculture towards more eco-friendly agricultural practices. Furthermore, in our model, we considered a unique production function and fixed prices and costs: we could refine it by distinguishing conventional and organic production methods. 


\section{A After Migration Biodiversity at the Stationary State}

\section{Proof of the Existence and the Uniqueness of $\tilde{B}_{i}^{\infty *}$ (Proposition 1)}

Let $z:=b_{i} K_{i}$. The internal stationary states, $\tilde{B}_{i}^{\infty *}$, of the system are the solutions of

$$
a-z x^{\theta}=x .
$$

This is equivalent to find the zeros of $f(x):=a-z x^{\theta}-x$. Note that it's a decreasing function of $x$ with $f(0)>0, \lim _{x \rightarrow \infty} f(x)<0$, and if $0<a-z<1, f(1)<0$. Then the biodiversity dynamics admits a unique positive internal stationary state $\tilde{B}_{i}^{\infty *}$ which is small than 1 if $a-z<1$. This proves Proposition 1 ,

\section{The Behavior of $\tilde{B}_{i}^{\infty *}$ w.r.t. the Parameters of the Model and Proof of Proposition 2}

By deriving the two sides of equation (16), then we can obtain:

- the derivative with respect to $a$ :

$$
\frac{\partial x}{\partial a}=\frac{1}{z \theta x^{\theta-1}+1}>0
$$

- the derivative with respect to $z$ is

$$
\frac{\partial x}{\partial z}=-\frac{x^{\theta}}{z \theta x^{\theta-1}+1}<0
$$

From the definition of $z$ and the definition of $K_{i}$, we obtain the behavior of $x$ w.r.t. the other parameters of the model. This prove the remark.

- the derivative with respect to $\theta$ is

$$
\frac{\partial x}{\partial \theta}=-\frac{z \theta \ln (x)}{z \theta x^{\theta-1}+1} .
$$

From the definition of $\theta$, we obtain the behavior of $x$ w.r.t. the other parameters of the model. From the definition of $\theta$, this prove the first inequality of Proposition 2 .

- We have that:

$$
\frac{\partial v(x)}{\partial \gamma}=x^{\gamma}\left(\ln (x)+\frac{\gamma\left(\frac{\partial x}{\partial \gamma}\right)}{x}\right)
$$




\section{B Proofs of Remark 2}

(i) When $N=2$, from the definition of $\tilde{B}_{i}$, we have that:

$$
\left\{\begin{array}{l}
\tilde{B}_{1}=m_{11} B_{1}+m_{12} B_{2} \\
\tilde{B}_{2}=m_{22} B_{2}+m_{21} B_{1}
\end{array}\right.
$$

which leads to:

$$
B_{j}=\frac{\tilde{B}_{j}-m_{j i} B_{1}}{m_{j j}} \Rightarrow \tilde{B}_{i}=m_{i i} B_{1}+m_{i j} \frac{\tilde{B}_{j}-m_{j i} B_{i}}{m_{j j}}, \quad i \neq j \in\{1,2\}
$$

we then obtain:

$$
B_{i}=\frac{m_{j} j \tilde{B}_{i}-m_{i j} \tilde{B}_{j}}{m_{i i} m_{j j}-m_{i j} m_{j i}}
$$

(ii) When the migration coefficient is the same, we can easily compute $B_{i}^{\infty}$. In fact, from the definition of (11), by imposing that:

$$
\left\{\begin{array}{l}
(1-m) B_{1}^{\infty *}+\frac{m}{N-1} \sum_{j \neq 1} B_{j}^{\infty *}=\tilde{B}_{1}^{\infty *} \\
\vdots \\
(1-m) B_{N}^{\infty *}+\frac{m}{N-1} \sum_{j \neq N} B_{j}^{\infty *}=\tilde{B}_{N}^{\infty *}
\end{array}\right.
$$

we obtain

$$
(1-m) B_{i}+\frac{m}{N-1}\left(\sum_{j=1}^{N} \tilde{B}_{j}-B_{i}=\tilde{B}_{i}, \quad \forall i=1 \ldots, N\right.
$$

and thus:

$$
\begin{aligned}
B_{i}^{\infty *} & =\frac{N-1}{N(1-m)-1}\left(\tilde{B}_{i}^{\infty *}-\frac{m}{N-1} \sum_{j=1}^{N} \tilde{B}_{j}^{\infty *}\right) \\
& =\frac{N-1}{N(1-m)-1}\left(\left(1-\frac{m}{N-1}\right) \tilde{B}_{i}^{\infty *}-\frac{m}{N-1} \sum_{j \neq i} \tilde{B}_{j}^{\infty *}\right) \\
& =\frac{1}{N(1-m)-1}\left((N-1-m) \tilde{B}_{i}^{\infty *}-m \sum_{j \neq i} \tilde{B}_{j}^{\infty *}\right) .
\end{aligned}
$$

(iii) If the farmers are symmetric, i.e. $a_{i}=a, b_{i}=b, \beta_{i}=\beta, \forall i=1, \ldots, N$, then we have: 
$B_{i}^{\infty *}=B_{j}^{\infty *}, \forall i, j=1, \ldots, N$. From this and (B) , then:

$$
\tilde{B}_{i}^{\infty *}=(1-m) B_{i}^{\infty *}+\frac{m}{N-1} \sum_{j \neq i} B_{j}^{\infty *}=B_{i}^{\infty *}
$$

and thus:

$$
B_{i}^{\infty *}=\tilde{B}_{i}^{\infty *}=B^{\infty *}, \quad \forall i=1 \ldots N
$$

\section{The Effects of Tax $\tau$}

\section{C.1 Proof of Proposition 4- Tax and $\tilde{B}_{i}^{\infty}$}

From equation (16), by deriving w.r.t. $\tau$, we obtain:

$$
\frac{\partial x}{\partial \tau}=-\frac{\partial z}{\partial \tau} x^{\theta}-z \theta x^{\theta-1} \frac{\partial x}{\partial \tau},
$$

which leads to:

$$
\frac{\partial x}{\partial \tau}=-\frac{\theta}{1+\theta z x^{\theta-1}} \frac{\partial z}{\partial \tau} x^{\theta} .
$$

Since

$$
\frac{\partial z}{\partial \tau}=\frac{\partial b_{i} K_{i}}{\partial \tau}=b_{i} \frac{\partial K_{i}}{\partial \tau}
$$

Since, from (21) we have that $\frac{\partial K_{i}}{\partial \tau}<0$ which leads to:

$$
\frac{\partial x}{\partial \tau}>0
$$

C.2 Proof of Proposition [5 - Tax and $B_{i}^{\infty}$

- Case $\mathbf{N}=\mathbf{2}$ From (12), we have:

$$
\frac{\partial B_{i}^{\infty *}}{\partial \tau}=\frac{1}{m_{i i} m_{j j}-m_{i j} m_{j i}}\left(m_{i j} \frac{\partial \tilde{B}_{i}}{\partial \tau}-m_{j j} \frac{\partial \tilde{B}_{j}}{\partial \tau}\right),
$$

and thus:

$$
\frac{\partial B_{i}^{\infty *}}{\partial \tau}>0 \Longleftrightarrow \frac{\partial \tilde{B}_{i}}{\partial \tau}>\frac{m_{j j}}{m_{i j}} \frac{\partial \tilde{B}_{j}}{\partial \tau}
$$


- Same migration coefficient From (13) we have:

$$
\frac{\partial B_{i}^{\infty *}}{\partial \tau}=\frac{\partial}{\partial \tau}\left[\frac{1}{N(1-m)-1}\left((N-1-m) \tilde{B}_{i}-m \sum_{j \neq i} \tilde{B}_{j}\right)\right],
$$

and thus, if $N(1-m)-1>0$ :

$$
\frac{\partial B_{i}^{\infty *}}{\partial \tau}>0 \Longleftrightarrow \frac{\partial \tilde{B}_{i}^{\infty *}}{\partial \tau}>\frac{m}{N-1-m} \sum_{j \neq i} \frac{\partial \tilde{B}_{j}^{\infty *}}{\partial \tau}
$$

\section{C.3 Proof of Proposition 6 - Tax and Optimal Solutions}

In order to prove Proposition [6, we first study the behavior of the coefficients $K_{A_{i}}, K_{\ell_{i}}, K_{Y_{i}}, K_{\pi_{i}}$ w.r.t. the $\operatorname{tax} \tau$.

- Tax and $K_{A_{i}}$

$$
\frac{\partial K_{A_{i}}}{\partial \tau}=\frac{\nu_{i}-1}{1-\eta_{i}-\nu_{i}}\left(w_{A}+\tau\right)^{\frac{2\left(\nu_{i}-1\right)+\eta_{i}}{1-\eta_{i}-\nu_{i}}}\left[p \beta_{i} \eta_{i}^{1-\nu_{i}} \nu_{i}^{\nu_{i}} w_{\ell}^{-\nu_{i}}\right]^{\frac{1}{1-\eta_{i}-\nu_{i}}}<0
$$

- Tax and $K_{\ell_{i}}$ :

$$
\frac{\partial K_{\ell_{i}}}{\partial \tau}=\frac{-\nu_{i}}{1-\eta_{i}-\nu_{i}}\left(w_{A}+\tau\right)^{\frac{\eta_{i}-1}{1-\eta_{i}-\nu_{i}}}\left[p \beta_{i} \eta_{i}^{\eta_{i}} \nu_{i}^{1-\eta_{i}}+w_{\ell}^{\eta_{i}-1}\right]<0
$$

- Tax and $K_{Y_{i}}$ :

$$
\frac{\partial K_{Y_{i}}}{\partial \tau}=\frac{-\nu_{i}}{1-\eta_{i}-\nu_{i}}\left[p^{\eta_{i}+\nu_{i}} \beta_{i} \eta_{i}^{\eta_{i}} \nu_{i}^{\nu_{i}}\left(w_{A}+\tau\right)^{-\eta-1} w_{\ell}^{-\nu_{i}}\right]^{\frac{1}{1-\eta_{i}-\nu_{i}}}<0
$$

- From previous inequalities, it follows that:

$$
\frac{\partial K_{i}}{\partial \tau}<0
$$

$$
\frac{\partial K_{\pi_{i}}}{\partial \tau}=p \frac{\partial K_{Y_{i}}}{\partial \tau}-K_{A_{i}}-\left(w_{A}+\tau\right) \frac{\partial K_{A_{i}}}{\partial \tau}-w_{\ell} \frac{\partial K_{\ell_{i}}}{\partial \tau}
$$

In this case the sign is not fixed (first term is negative, the others are positive), i.e. the behavior of the profit w.r.t. the tax is not fixed. 
From the previous inequalities we can prove that the behavior of the optimal solutions w.r.t. the tax is not fixed:

- Yield $Y_{i}^{\infty *}$ and tax:

$$
\begin{aligned}
\frac{\partial Y_{i}^{\infty *}}{\partial \tau} & =\frac{\partial K_{Y_{i}}}{\partial \tau} \tilde{B}^{\theta}+K_{Y_{i}} \theta \tilde{B}^{\theta-1} \frac{\partial \tilde{B}}{\partial \tau} \\
& =\frac{\partial K_{Y_{i}}}{\partial \tau} \tilde{B}^{\theta}+K_{Y_{i}} \theta \tilde{B}^{\theta-1} \frac{\partial \tilde{B}}{\partial K_{i}} \frac{\partial K_{i}}{\partial \tau}
\end{aligned}
$$

From $₫ \frac{\partial \tilde{B}}{\partial K_{i}}=-\frac{b \tilde{B}^{\theta}}{b_{i} \theta K_{i} \tilde{B}^{\theta-1}+1}$.

If $\alpha=0$, then $K_{i}=K_{Y_{i}}$ and thus, in this case, we obtain:

$$
\left.\frac{\partial Y_{i}^{\infty *}}{\partial \tau}=\frac{\partial K_{i}}{\partial \tau} \tilde{B}^{\theta}\right)\left(1-K_{Y_{i}} \theta \tilde{B}^{\theta-1} \frac{b \tilde{B}^{\theta-1}}{b_{i} \theta \tilde{B}^{\theta-1}+1}\right)
$$

We now that $\frac{\partial K_{i}}{\partial \tau}<0$. Let $K_{i} \theta b_{i} \tilde{B}^{\theta-1}=z$. We have that:

$$
1-\frac{z}{z+1}>0 \forall z>-1 \Rightarrow \text { If } \alpha=0, \text { then } \frac{\partial K_{i}}{\partial \tau}<0
$$

- Inputs $A_{i}^{\infty *}$ and tax:

$$
\begin{aligned}
& \frac{\partial A_{i}^{\infty *}}{\partial \tau}=\frac{\partial K_{A_{i}}}{\partial \tau} \tilde{B}^{\theta}+K_{A_{i}} \theta \tilde{B}^{\theta-1} \frac{\partial \tilde{B}}{\partial \tau}= \\
& =\tilde{B}^{\theta}\left(p \beta_{i} \eta^{1-\nu} \nu^{\nu}\left(w_{A}+\tau\right)^{(\nu-1)} w_{\ell}^{-\nu}\right)^{\frac{1}{1-\eta-\nu}}\left(\frac{\nu-1}{1-\eta-\nu}\left(w_{A}+\tau\right)^{-1}+\theta \tilde{B}^{-1} \frac{\partial \tilde{B}}{\partial \tau}\right) .
\end{aligned}
$$

And thus:

$$
\frac{\partial A_{i}^{\infty *}}{\partial \tau}>0 \Longleftrightarrow \frac{\partial \tilde{B}}{\partial \tau}<\frac{1-\nu}{\gamma} \frac{\tilde{B}}{w_{A}+\tau}
$$




\section{Bibliography}

\section{References}

(2005). Ecosystems and human well-being: synthesis. Millennium Ecosystem Assessment, Island Press, Washington, DC.

Bluthgena, N., Dormannb, C., Pratic, D., Klausd, V., Kleinebeckerd, T., Holzeld, N., Alte, F., Bochc, S., Gockelf, S., Hempg, A., Mullerg, J., Nieschulzeh, J., Renneri, S., Schoningh, I., Schumacherg, U., Socherc, S., Wellsi, K., Birkhoferj, K., Buscotk, F., Oelmanne, Y., Rothenwohrerm, C., Scherberm, C., Tscharntkem, T., Weinern, C., Fischerc, M., Kalkoi, E., Linsenmairn, K., Schulzeh, E., and Weisser, W. (2012). A quantitative index of land-use intensity in grasslands: Integrating mowing, grazing and fertilization. Basic and Applied Ecology, 13:207-220.

Butchart, S., Walpole, M., Collen, B., van Strien, A., and et al., J. S. (2010). Global biodiversity: indicators of recent declines. Science, 328:1164-1168.

Cordonnier, T. and Peyron, J.-L. (2015). Reconciling environment and production in managed ecosystems: Is ecological intensification a solution? Environmental Management, 56:1035-1038.

Desquilbet, M., Dorin, B., and Couvet, D. (2013). Land sharing vs land sparing for biodiversity: How agricultural markets make the difference. Innovations Agronomiques, 32:377-389.

Durand, M., Désilles, A., P.Saint-Pierre, Angeon, V., and Ozier-Lafontaine, H. (2017). Agroecological transition: A viability model to assess soil restoration? Nat. Resour. Model., 30:e12134.

Garibaldi, L., Aizena, M., Kleinc, A., Cunninghamd, S. A., and Hardere, L. (2011). Global growth and stability of agricultural yield decrease with pollinator dependence. In Proc. Natl. Acad. Sci. USA, volume 14, pages 5909-5914.

Geertsema, W., Rossing, W., Landis, D., Bianchi, F., van Rijn, P. C., Schaminee, J. H., Tscharntke, T., and van der Werf, W. (2016). Actionable knowledge for ecological intensification of agriculture. Frontiers in Ecology and the Environment, 14:209-2016.

Mouysset, L., Doyen, L., and Jiguet, F. (2013). How does economic risk adversion affect biodiversity? Ecological Applications, 23:96-109.

Newbold, T., Hudson, L., Hill, S., Contu, S., and I.Lysenko (2015). Global effects of land use on local terrestrial biodiversity. Nature, 520:45-50. 
Nishimura, K. and Kishida, O. (2001). Coupling of two competitive systems via density dependent migration. Ecological Research, 16:359-368. 


\section{CEE-M Working Papers - 2018}

WP 2018 - 01: $\quad$ Ilaria Brunetti., Mabbel Tidball, $\&$ Denis Couvet

« Relationship Between Biodiversity and Agricultural Production » 